\title{
Origin of multiple intersubunit rotations before EF-G-catalyzed ribosomal translocation through the mRNA with a downstream secondary structure
}

Ping Xie

\begin{abstract}
Background: A ribosome can translate through both the single-stranded region and duplex region of messenger RNA (mRNA). Recent single molecule fluorescence energy transfer (smFRET) data showed that when the ribosome translates through the mRNA containing a downstream stem loop, the ribosomal complex exhibits multiple fluctuations between the classical non-rotated and hybrid states before undergoing translocation in the presence of elongation factor $G$ and GTP (EF-G.GTP) of high concentration; by contrast, when the ribosome translates through the mRNA lacking the stem loop, the complex samples the hybrid state approximately once before undergoing translocation.

Results: Based on our proposed model, we provide theoretical analyses of the dynamics of the multiple fluctuations before undergoing mRNA translocation, providing quantitative explanations of the smFRET data.

Conclusions: The good quantitative agreement between the theoretical data and the smFRET data supports the model showing that at saturating EF-G.GTP the multiple fluctuations with the mRNA containing the stem loop occur with EF-G still bound to the ribosome, rather than occur after the release of EF-G from the hybrid state and before the re-binding of EF-G; the multiple fluctuations are induced by occurrences of the futile transition during the mRNA translocation, which is induced by the resistance force resulting from the unwinding of the downstream mRNA stem loop to impede the ribosomal translocation; by contrast, with the mRNA lacking the stem loop, no resistance is present to impede the translocation and thus, no futile transition occurs, giving the ribosomal complex sampling the hybrid state once before undergoing translocation.
\end{abstract}

Keywords: Ribosome, Classical non-rotated state, Hybrid state, tRNA translocation, mRNA translocation, Translation

\section{Background}

The ribosome is a macromolecular machine that is responsible for the synthesis of protein chains by a process called translation, where the sequence of the amino acid residues is encoded by that of codons (triplets of nucleotides) on a single-stranded messenger RNA (mRNA) [1-6]. It was characterized that the ribosome can also translate through the mRNA duplex or secondary structure by unwinding the duplex or secondary structure $[7,8]$. Thus, elucidating the molecular mechanism and

\footnotetext{
Correspondence: pxie@aphy.iphy.ac.cn
}

Key Laboratory of Soft Matter Physics and Beijing National Laboratory for Condensed Matter Physics, Institute of Physics, Chinese Academy of Sciences, Beijing 100190, China dynamics of ribosome translation through the mRNA secondary structure is an active area of investigation. Using optical trapping technique, Qu et al. [9] studied the dynamics of ribosome translation through the duplex region of mRNA under the effect of the external force to unzip the duplex, where it was shown interestingly that the translation rate has a sigmoid dependence on the external force. Recently, using single molecule fluorescence energy transfer (smFRET), Chen et al. [10] studied the effect of downstream mRNA secondary structures on tRNA translocation in the large $50 \mathrm{~S}$ subunit and deacylated tRNA dissociation from the $\mathrm{E}$ site, where it was found that the downstream mRNA secondary structures have a more sensitive effect on the deacylated tRNA 
dissociation than on the tRNA translocation in the $50 \mathrm{~S}$ subunit.

More recently, using smFRET between the Cy3labeled L1 stalk of the 50S subunit and a Cy5-labeled tRNA in the ribosomal peptidyl-tRNA-binding (P) site, Kim et al. [11] studied the dynamics of ribosomal translocation through an mRNA containing a downstream secondary structure, a frameshifting stimulatory stem loop, positioned at the ribosomal mRNA entry channel. Similar to that observed by Chen et al. [10], Kim et al. [11] also observed significantly slower deacylated tRNA dissociation with the mRNA containing the downstream stem loop in comparison with an mRNA lacking the stem loop. More interestingly, it was observed that the P-site tRNA/L1 stalk of the pretranslocation ribosomal complexes bound with the mRNA containing the stem loop exhibits multiple fluctuations between the classical non-rotated and hybrid states at high concentration of elongation factor $\mathrm{G}$ (EF-G) before undergoing translocation, in contrast with the complexes bound with the mRNA lacking the stem loop, which samples the hybrid state approximately once before undergoing translocation. Even at saturating concentration of EF-G.GTP $(1 \mu \mathrm{M})$ (the smFRET data with the mRNA lacking the stem loop showed that the translocation time decreases with increasing EF-G.GTP concentration up to $0.5 \mu \mathrm{M}$ and levels), about $50 \%$ of the traces with the mRNA containing the stem loop are still fluctuating.

To explain the multiple fluctuations between the classical non-rotated and rotated (or hybrid) conformations occurring before ribosomal translocation, Kim et al. [11] proposed the presence of an intermediate state (State I) between the hybrid state bound with EF-G (State G) and the posttranslocation state (State Post) (see Additional file 1: Figure S1). For the mRNA containing the stem loop (FSmRNA), the energy barrier of transition from State G to State I was proposed to become much higher than the energy barriers of transitions between State G and the hybrid state without EF-G (State $\mathrm{H}$ ) and those between State $\mathrm{H}$ and the classical non-rotated state (State C). Since from this proposal it is deduced that even at saturating concentration of EF-G the number of the transition from the hybrid state to the classical nonrotated state is also sensitively dependent on EF-G concentration, which is inconsistent with the deduction from the smFRET data [11] (see Discussion for detail), it is likely improbable that the multiple fluctuations can only occur after the release of EF-G. Thus, it is unclear whether the multiple fluctuations can only occur after the release of EF-G or can also occur with EF-G still bound to the ribosome. If the multiple fluctuations can occur with EF-G still bound to the ribosome, what is the molecular mechanism that the downstream stem loop induces such fluctuations? Why are such multiple fluctuations observed only with the mRNA containing the stem loop? Why do the ribosomal complexes sample the hybrid state once with the mRNA lacking the stem loop?

The purpose of this work is to address these unclear issues and provide quantitative explanations of the smFRET data of Kim et al. [11]. Based on our explanations we propose that the observed multiple fluctuations between the classical non-rotated and rotated (or hybrid) conformations can occur with EF-G still bound to the ribosome rather than occur after EF-G release and the multiple fluctuations result from the futile transition occurring during the mRNA translocation. The determination of the origin of the multiple fluctuations before mRNA translocation is critical to the molecular mechanism of ribosome translation through the mRNA secondary structure.

\section{Methods}

\section{The model}

First we describe the model of ribosomal translocation through the mRNA containing a downstream mRNA secondary structure (Figure 1) [12]. To be consistent with the available structural data [13], we consider that there are at least two hybrid states bound with EF-G, called State H and State H1. In the two hybrid states, deacylated tRNA locates in the nearly same position of the 50S E site while the peptidyl-tRNA moiety locates in different positions of the $50 \mathrm{~S}$ subunit. In State H1, the ribosomal unlocking can occur, widening the mRNA channel and then facilitating the reverse ribosomal rotation. Here, for the sake of conciseness, we consider saturating concentration of EF-G.GTP (for the model and studies at non-saturating EF-G.GTP concentration, see Additional file 1: Figure S2 and Text S1).

We begin with the peptidyl-tRNA in the P site and the aminoacyl-tRNA in the ribosomal aminoacyl-tRNAbinding (A) site (State I), which is followed by the peptidyl transfer, with the deacylated tRNA in the P site and the peptidyl-tRNA in the A site (State C0). Then, EF-G. GTP at saturating concentration binds immediately to State C0, becoming State C. State C transits to State H and then to State H1, where the ribosomal unlocking occurs (State H2), widening the mRNA channel. The subsequent rapid reverse ribosomal rotation can induce the transition of State H2 either to State POST (with a probability $P_{E}$ ) or to State $\mathrm{F} 1$ (with a probability $1-P_{E}$ ), which is also accompanied by the rapid Pi release. The origin of the two transitions induced by the reverse ribosomal rotation is stated as follows.

If no downstream mRNA secondary structure is present, the reverse ribosomal rotation drives the two tRNAs coupled with the mRNA to move from the small 30S P and A sites to the 30S E and P sites, respectively, while the two tRNAs are kept fixed to the large $50 \mathrm{~S} \mathrm{E}$ 


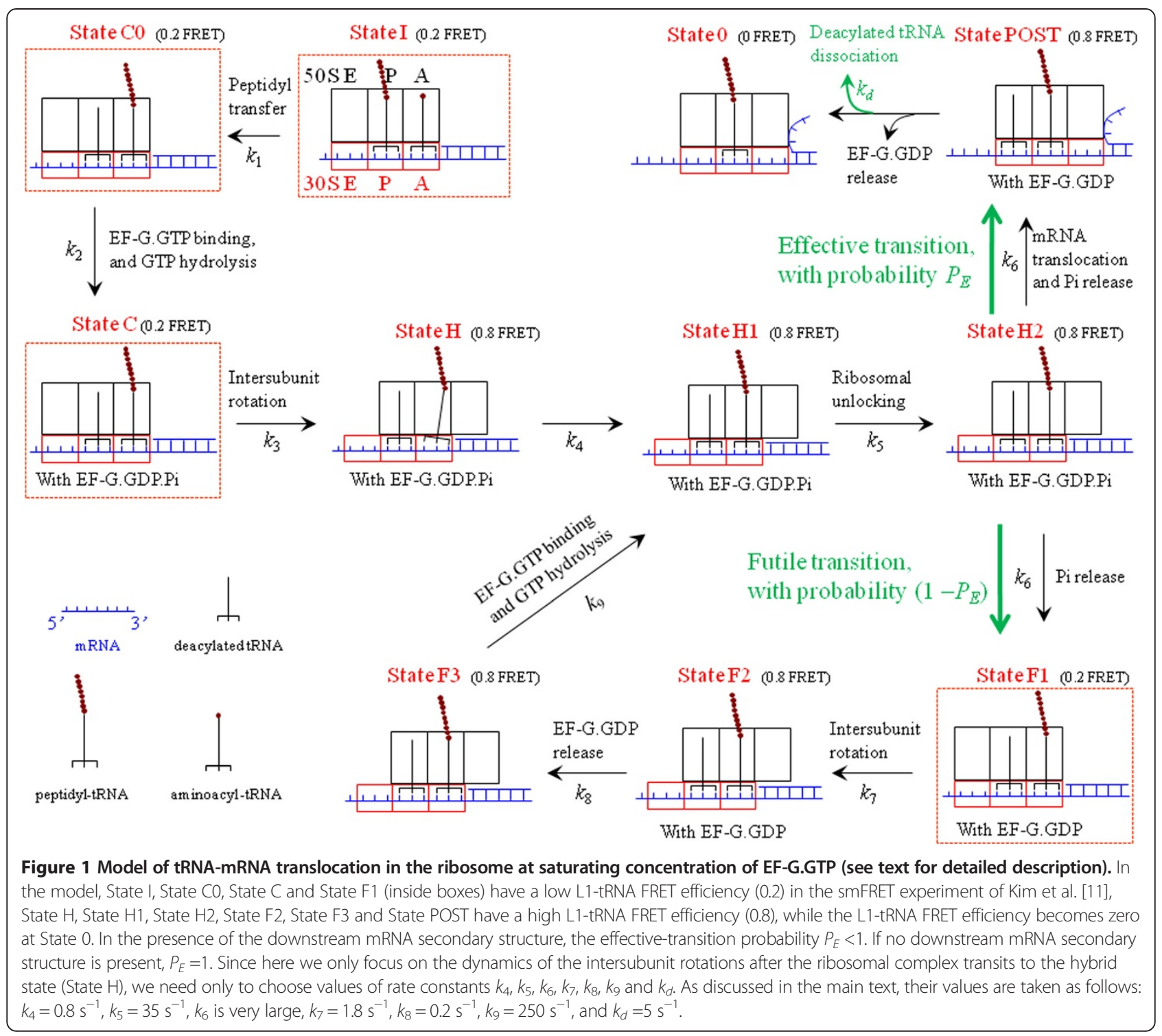

and $\mathrm{P}$ sites due to the high affinity $\left(E_{P E}^{(50 S)}\right)$ of the $50 \mathrm{~S} \mathrm{E}$ and P sites for the deacylated tRNA [14] and the peptidyl-tRNA [15], respectively. This induces the transition of State $\mathrm{H} 2$ to State POST, which is called effective transition. On the contrary, if the downstream mRNA secondary structure of a very strong stability is present, the downstream mRNA structure resists the $30 \mathrm{~S}$ subunit to move relative to the mRNA that is coupled with the two tRNAs via codon-anticodon interaction. Thus, the reverse ribosomal rotation drives the $50 \mathrm{~S}$ subunit to move relative to the two tRNAs by overcoming the finite affinity $\left(E_{P E}^{(50 S)}\right)$ of the $50 \mathrm{~S} \mathrm{E}$ and $\mathrm{P}$ sites for the two tRNAs. This induces the transition of State $\mathrm{H} 2$ to State F1, which is called futile transition. Thus, for the real case of the downstream mRNA secondary structure of an intermediate stability, the reverse ribosomal rotation can induce either the effective transition by unwinding three mRNA base pairs or the futile transition by overcoming the binding energy $E_{P E}^{(50 S)}$ but without unwinding of the mRNA base pairs, with the probability of effective transition, $P_{E}$, depending on the stability of the mRNA secondary structure.

After the ribosomal complex transits from the rotated conformation (State H2) to the non-rotated conformation (either State POST or State F1), the mRNA channel in the 30S subunit becomes tight again, as proposed by Frank and Agrawal [16]. Note that both State POST and State F1 are bound with EF-G.GDP. In State POST, the ribosome becomes relocked and EF-G.GDP is then released (State 0). State F1 is in the classical non-rotated pretranslocation conformation. As EF-G.GDP facilitates the transition to and stabilizes the hybrid state of the pretranslocation ribosomal complex $[17,18]$, as discussed 
in detail before [12], State F1 then transits to State F2, from which EF-G.GDP is released (State F3) ${ }^{\mathrm{a}}$. After the binding of EF-G.GTP and then rapid GTP hydrolysis, State F3 becomes State H1 again, from which the transition to State $\mathrm{H} 2$ proceeds, as just mentioned above.

It is noted here that since State POST is the posttranslocation state while State F2 is the pretranslocation state, the rate of EF-G.GDP release from State POST is different from that from State F2 [19]. In addition, it is noted that State I, State C0, State C and State F1 (inside boxes) have a low L1-tRNA FRET efficiency (0.2) in the smFRET experiment of Kim et al. [11], State H, State H1, State H2, State F2, State F3 and State POST have a high L1-tRNA FRET efficiency (0.8), while State 0 has a zero L1-tRNA FRET efficiency.

As mentioned above, for the case that no downstream mRNA secondary structure is present, the transitions can still be described by Figure 1, but with the probability of effective transition, $P_{E}=1$, i.e., without the occurrence of the futile transition.

\section{The simulation method}

Based on the pathway (Figure 1), we use Monte Carlo algorithm to simulate the time trace of FRET efficiency, as used elsewhere [20]. In our simulations, during each time step $\Delta t\left(\Delta t=10^{-5} \mathrm{~s}\right.$ in our simulations), a random number $x$ is generated with uniform probability between 0 and 1 . If $x>P_{i}$, the transition of rate $k_{i}(i=1, \ldots, 9$ and d) occurs; if $x>P_{i}$, the transition of rate $k_{i}$ does not occur, where $P_{i}=k_{i} \Delta t$ is the probability of state transition. Moreover, as mentioned above, the L1-tRNA FRET efficiency is set to be 0.2 in State I, State C0, State C and State F1, the L1-tRNA FRET efficiency is set to be 0.8 in State H, State H1, State H2, State F2, State F3 and State POST, and the L1-tRNA FRET efficiency is set to be 0 in State 0.

Based on the pathway (Figure 1), we make use of analytical solutions to study the time distributions for intersubunit rotations occurring before translocation through the mRNAs containing and/or lacking the downstream secondary structures (for detail, see Additional file 1: Text S2).

\section{Results}

\section{Choice of parameter values}

In this work, since we only focus on the dynamics of the intersubunit rotations after the ribosomal complex transits to the hybrid state (State $\mathrm{H}$ ), we need only to choose values of rate constants $k_{4}, k_{5}, k_{6}, k_{7}, k_{8}, k_{9}$ and $k_{d}$. The available biochemical data showed that the ribosomal unlocking rate is about $35 \mathrm{~s}^{-1}$ and after the unlocking the mRNA translocation or Pi release is fast [21]. Thus, we take $k_{5}=35 \mathrm{~s}^{-1}$ and $k_{6}$ has a large value in the calculation. After EF-G.GTP binds to the ribosome, the rate of GTP hydrolysis is about $250 \mathrm{~s}^{-1}$ [21]. Thus, we take $k_{9}=250 \mathrm{~s}^{-1}$ in the calculation. By fitting to the single molecule experimental data of Uemura et al. [22], it is obtained that the rate of deacylated tRNA dissociation from the posttranslocation state is about $5 \mathrm{~s}^{-1}$ [23]. Thus, we take $k_{d}=5 \mathrm{~s}^{-1}$ in the calculation. The recent single molecule data showed that the mean time of EFG.GDP bound to the rotated hybrid pretranslocation state is about 20-fold longer than that of EF-G.GDP bound to the non-rotated posttranslocation state [19]. On the other hand, the biochemical data showed that after transition to the posttranslocation state, the ribosome becomes relocked with a rate of about $5 \mathrm{~s}^{-1}$, which is followed by the release of EF-G.GDP with a rate of about $20 \mathrm{~s}^{-1}$ [2], giving the release rate of EF-G.GDP from the posttranslocation state to be about $4 \mathrm{~s}^{-1}$. Thus, we take the release rate of EF-G.GDP from the rotated hybrid pretranslocation state, $k_{8}=0.2 \mathrm{~s}^{-1}$, in the calculation. Moreover, we take $k_{7}=1.8 \mathrm{~s}^{-1}$, which is consistent with the smFRET data [24-26] on the EF-G-facilitating forward ribosomal rotation. Thus, of the seven rate constants $k_{4}, k_{5}, k_{6}, k_{7}, k_{8}, k_{9}$ and $k_{d}$, only one rate constant $k_{4}$ is adjustable. As it will be seen below, by taking $k_{4}=$ $0.8 \mathrm{~s}^{-1}$, the theoretical data are consistent with the smFRET data of Kim et al. [11]. Thus, throughout we take $k_{4}=0.8 \mathrm{~s}^{-1}$.

In addition, to be consistent with the smFRET data showing that about $50 \%$ of the traces for the mRNA containing the stem loop were fluctuating at saturating concentration of EF-G.GTP [11], we take $P_{E}=0.5$ throughout our calculation for the mRNA containing

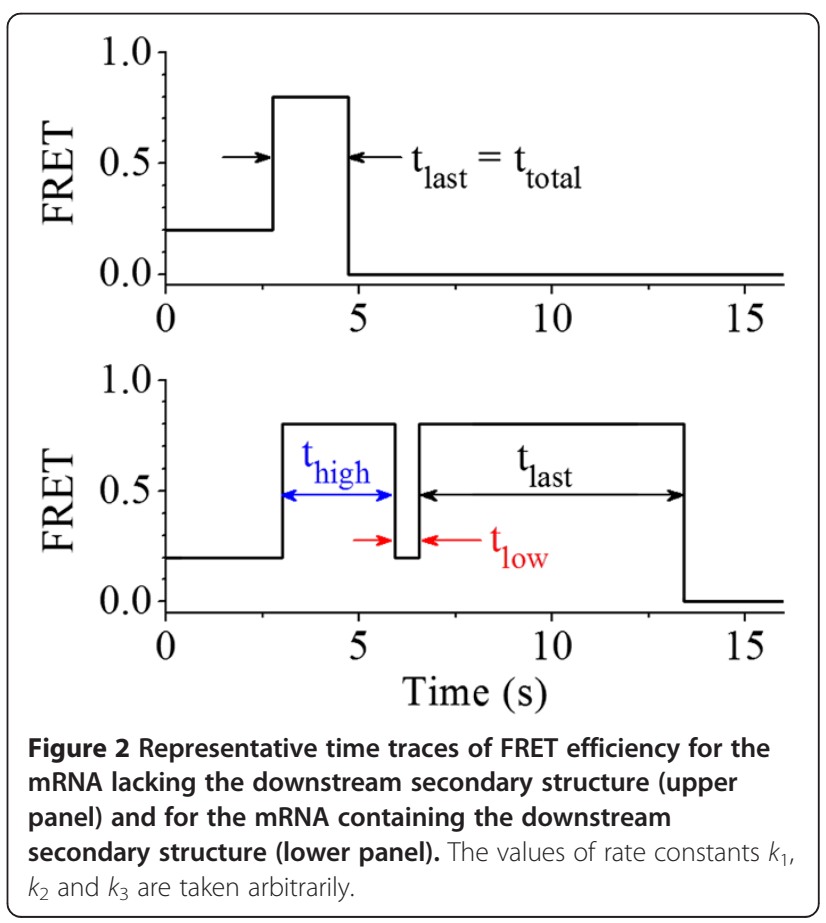




\section{$\mathrm{H} \stackrel{k_{4}}{\longrightarrow} \mathrm{H} 1 \stackrel{k_{5}}{\longrightarrow} \mathrm{H} 2 \stackrel{k_{6}}{\longrightarrow} \mathrm{POST} \stackrel{k_{d}}{\longrightarrow} 0$}

Scheme 1 The transitions in the time period of the last or total high FRET efficiency for the mRNA lacking the downstream secondary structure.

the downstream secondary structure, which is close to the value obtained by fitting to the optical trapping data of Qu et al. [9] and that obtained by comparing with the smFRET data of Chen et al. [10] presented in the previous works [12,27]. For the mRNA lacking the stem loop, we take $P_{E}=1$, as mentioned above. It should be emphasized that for both the mRNA lacking the downstream secondary structure and the mRNA containing the downstream secondary structure, except that $P_{E}$ has different values the rate constants $k_{4}, k_{5}, k_{6}, k_{7}, k_{8}, k_{9}$ and $k_{d}$ have the same values.

\section{Dynamics of intersubunit rotations}

Using the Monte Carlo method, we simulate the time trace of FRET efficiency based on the pathway of Figure 1. In Figure 2, we show two representative time traces of the FRET efficiency with the mRNA lacking the downstream secondary structure (upper panel) and with the mRNA containing the downstream secondary structure (lower panel). The simulated traces shown in Figure 2 resemble the smFRET data (Figure Two(B) in Kim et al. [11]). As done in Kim et al. [11], in Figure 2 we define some time periods: $t_{\text {high }}, t_{\text {low }}, t_{\text {last }}$ and $t_{\text {total }}$.

\section{Time distributions for the mRNA lacking the downstream secondary structure}

For the mRNA lacking the downstream secondary structure (with $P_{E}=1$ ), as the ribosomal complex samples the hybrid state only once before undergoing translocation, the time distribution of the last high FRET efficiency $(0.8)$ is the same as that of the total high FRET efficiency, which is denoted by $f_{\Delta}(t)$. In the model (Figure 1 ), the time period of the last or total high FRET efficiency corresponds to the transitions of Scheme 1.

As $k_{6}$ is very fast, from Scheme 1 we can easily obtain $f_{\Delta}(t)$ having the form (see Additional file 1: Text S2)

$$
\begin{aligned}
f_{\Delta}(t)=k_{4} k_{5} k_{d}\left[\frac{\exp \left(-k_{4} t\right)}{\left(k_{4}-k_{5}\right)\left(k_{4}-k_{d}\right)}\right. & +\frac{\exp \left(-k_{5} t\right)}{\left(k_{5}-k_{4}\right)\left(k_{5}-k_{d}\right)} \\
& \left.+\frac{\exp \left(-k_{d} t\right)}{\left(k_{d}-k_{4}\right)\left(k_{d}-k_{5}\right)}\right]
\end{aligned}
$$

With Eq. (1), we calculate $f_{\Delta}(t)$, with the results shown in Figure 3, which are in good agreement with the
smFRET data (the left panel of Figure Two(F) in Kim et al. [11]).

\section{Time distributions for the mRNA containing the downstream secondary structure}

For the mRNA containing the downstream secondary structure (with $P_{E}<1$ ), from Figure 1 the time period of low FRET efficiency $\left(t_{\text {low }}\right)$ corresponds to the transition of Scheme 2.

From Scheme 2, the time distribution of low FRET efficiency, $f_{\text {low }}(t)$, has the from

$$
f_{\text {low }}(t)=k_{7} \exp \left(-k_{7} t\right)
$$

With Eq. (2), the calculated results of $f_{\text {low }}(t)$ are shown in Figure $4 \mathrm{a}$, which are in good agreement with the smFRET data (the bottom panel of Figure S7A in Kim et al. [11]).

From Figure 1, the time period of the last high FRET efficiency $\left(t_{\text {last }}\right)$ corresponds to the transitions of Scheme 3.

As $k_{6}$ is very fast and $k_{9}\left(250 \mathrm{~s}^{-1}\right)$ is much larger than $k_{8}\left(0.2 \mathrm{~s}^{-1}\right), k_{5}\left(35 \mathrm{~s}^{-1}\right)$ and $k_{d}\left(5 \mathrm{~s}^{-1}\right)$, from Scheme 3 we can easily obtain the time distribution of the last high FRET efficiency, $f_{\text {last }}(t)$, approximately having the form

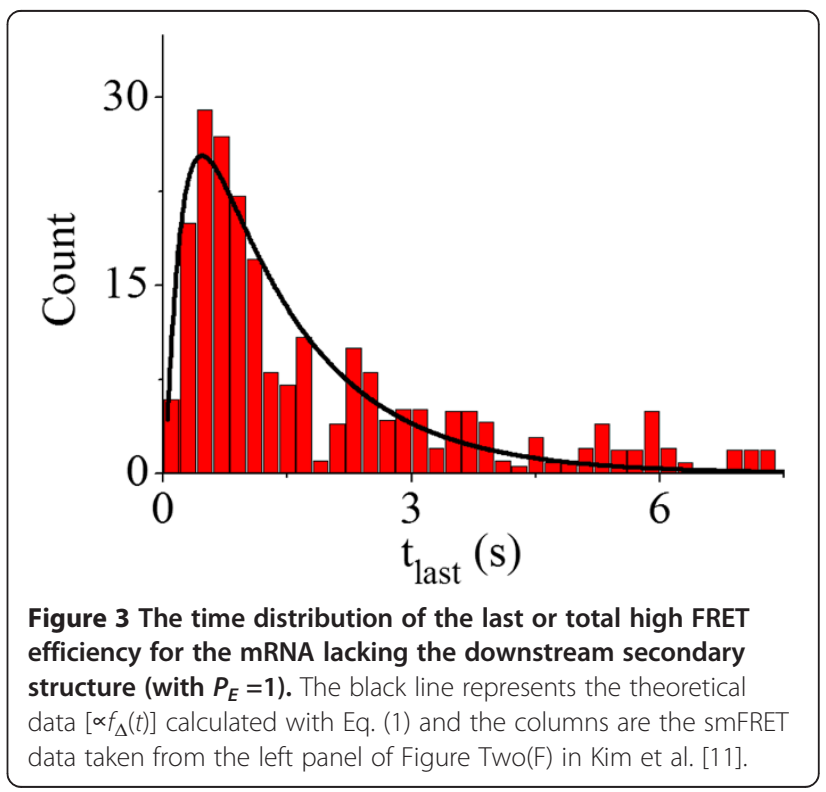




\section{$\mathrm{F} 1 \stackrel{k_{7}}{\longrightarrow} \mathrm{F} 2$}

Scheme 2 The transition in the time period of low FRET efficiency for the mRNA containing the downstream secondary structure.

$$
\begin{aligned}
f_{\text {last }}(t)=k_{8} k_{5} k_{d}\left[\frac{\exp \left(-k_{8} t\right)}{\left(k_{8}-k_{5}\right)\left(k_{8}-k_{d}\right)}\right. & +\frac{\exp \left(-k_{5} t\right)}{\left(k_{5}-k_{8}\right)\left(k_{5}-k_{d}\right)} \\
& \left.+\frac{\exp \left(-k_{d} t\right)}{\left(k_{d}-k_{8}\right)\left(k_{d}-k_{5}\right)}\right]
\end{aligned}
$$

With Eq. (3), the calculated results of $f_{\text {last }}(t)$ are shown in Figure $4 b$, which are consistent with the smFRET data (the right panel of Figure Two(F) in Kim et al. [11]).

Now, we study the time distribution of the high FRET efficiency $\left(t_{\text {high }}\right)$. As in the experiment [11], only the traces with the presence of the transition from the hybrid to non-rotated states before undergoing translocation are considered. Then, from Figure 1 we note that in the first round of intersubunit rotations, the time period of the high FRET efficiency corresponds to the transitions of Scheme 4. In the other rounds of intersubunit rotations, the time period of the high
FRET efficiency corresponds to the transitions of Scheme 5.

As $k_{6}$ is very fast, from Scheme 4 we can easily obtain that the time distribution of the high FRET efficiency in the first round of intersubunit rotations, $f_{H 1}(t)$, has the form

$$
f_{H 1}(t)=\frac{k_{4} k_{5}}{k_{5}-k_{4}}\left[\exp \left(-k_{4} t\right)-\exp \left(-k_{5} t\right)\right]
$$

As $k_{6}$ is very fast and $k_{9}$ is much larger than $k_{8}$ and $k_{5}$, from Scheme 5 the time distribution of the high FRET efficiency in the other rounds of intersubunit rotations, $f_{H 2}(t)$, approximately has the form

$$
f_{H 2}(t)=\frac{k_{8} k_{5}}{k_{5}-k_{8}}\left[\exp \left(-k_{8} t\right)-\exp \left(-k_{5} t\right)\right]
$$

The time distribution of the high FRET efficiency can be then calculated by $f_{H}(t)=f_{H 1}(t)+\sum_{n=1}^{\infty}\left(1-P_{E}\right)^{n} f_{H 2}(t)$, which is rewritten as $f_{H}(t)=f_{H 1}(t)+\left(1-P_{E}\right) / P_{E} f_{H 2}(t)$. With Eqs. (4) and (5), we have

$$
\begin{aligned}
f_{H}(t)= & \frac{k_{4} k_{5}}{k_{5}-k_{4}}\left[\exp \left(-k_{4} t\right)-\exp \left(-k_{5} t\right)\right] \\
& +\frac{1-P_{E}}{P_{E}} \frac{k_{8} k_{5}}{k_{5}-k_{8}}\left[\exp \left(-k_{8} t\right)-\exp \left(-k_{5} t\right)\right]
\end{aligned}
$$
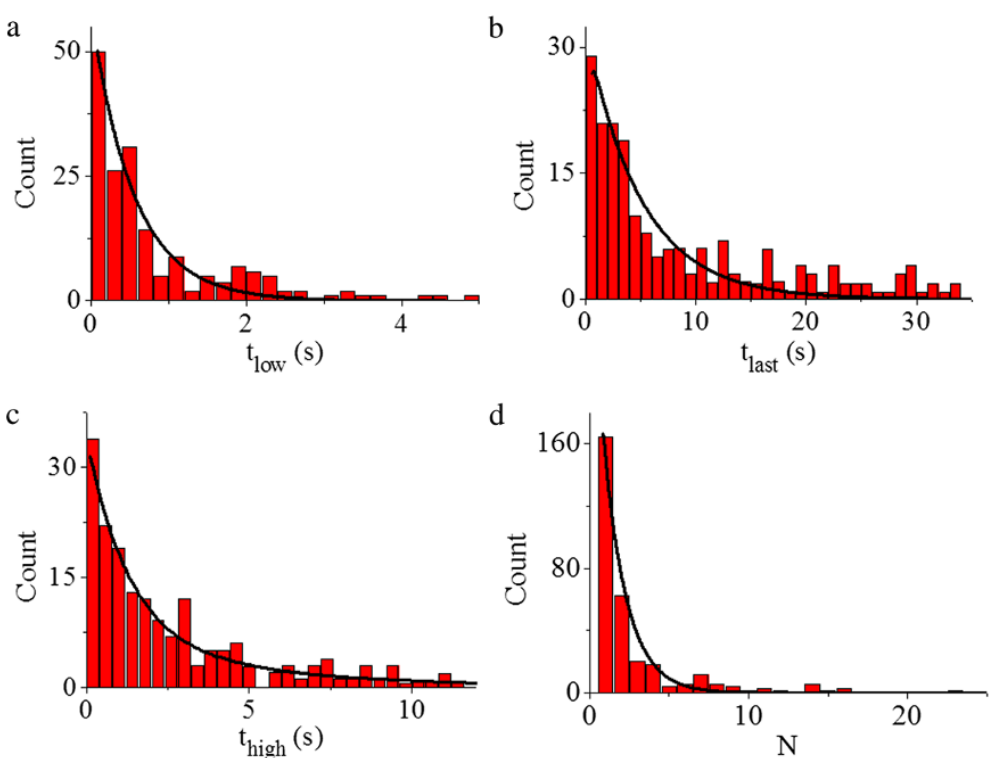

Figure 4 Results for the mRNA containing the downstream secondary structure (with $P_{E}=0.5$ ). (a) The time distribution of the low FRET efficiency, with the black line representing the theoretical data $\left[\alpha f_{\text {low }}(t)\right]$ calculated with Eq. (2) and the smFRET data (columns) being taken from the bottom panel of Figure S7A in Kim et al. [11]. (b) The time distribution of the last high FRET efficiency, with the black line representing the theoretical data $\left[\propto f_{\text {last }}(t)\right]$ calculated with Eq. (3) and the smFRET data (columns) being taken from the right panel of Figure two $\mathrm{F}$ in Kim et al. [11]. (c) The time distribution of the high FRET efficiency, with the black line representing the theoretical data $\left[\propto f_{H}(t)\right]$ calculated with Eq. (6) and the smFRET data (columns) being taken from the bottom panel of Figure S7B in Kim et al. [11]. (d) The distribution of the number ( $N$ ) of transitions to the high FRET efficiency per trace before the disappearance of the FRET efficiency, with the black line representing the theoretical data $[\propto f(N)]$ calculated with Eq. (7) and the smFRET data (columns) being taken from the bottom panel of Figure S7C in Kim et al. [11]. 


\section{$\mathrm{F} 2 \stackrel{k_{8}}{\longrightarrow} \mathrm{F} 3 \stackrel{k_{9}}{\longrightarrow} \mathrm{H} 1 \stackrel{k_{5}}{\longrightarrow} \mathrm{H} 2 \stackrel{k_{6}}{\longrightarrow} \mathrm{POST} \stackrel{k_{d}}{\longrightarrow} 0$}

Scheme 3 The transitions in the time period of the last high FRET efficiency for the mRNA containing the downstream secondary structure.

With Eq. (6), the calculated results of $f_{H}(t)$ are shown in Figure 4c, which are in good agreement with the smFRET data (the bottom panel of Figure S7B in Kim et al. [11]).

In the above we show the calculated results of the time distributions with values of the rate constant $k_{i}(i=4,6 \ldots$, 9 and $d$ ) given in the legend of Figure 1 . To see how the uncertainties of these values affect the results, we replace $k_{i}$ with $k_{i} \pm \Delta k_{i}$, where $\Delta k_{i}=0.1 k_{i}$. The calculated results (see Additional file 1: Figure S5) show that even with an about $10 \%$ uncertainty for each $k_{i}$, the theoretical results are still consistent with the smFRET data.

Finally, we study the distribution of the number $(N)$ of transitions to the high FRET efficiency per trace before the disappearance of the Cy5 signals. Based on the model (Figure 1), the distribution of the number, $f(N)$, can be calculated by

$$
f(N)=P_{E}\left(1-P_{E}\right)^{(N-1)}
$$

With Eq. (7), the calculated results of $f(N)$ are shown in Figure $4 d$, which are consistent with the smFRET data (the bottom panel of Figure S7C in Kim et al. [11]).

\section{Discussion}

It should be mentioned that in our analysis of the dynamics of intersubunit rotations we have not considered the dissociation of EF-G after it binds to the ribosome. In fact, in the model of Figure 1 even with the consideration that EF-G can be dissociated from any state before the ribosomal unlocking occurs, we have the nearly same results as we presented in this work, which is discussed as follows. For example, after EF-G is dissociated from State H, EF-G.GTP of saturating concentration would bind to State $\mathrm{H}$ immediately. Moreover, after EF-G.GTP binding to the ribosome, the rate of GTP hydrolysis $\left(250 \mathrm{~s}^{-1}\right)$ [21] is much higher than $k_{4}\left(0.8 \mathrm{~s}^{-1}\right)$. Thus, the rebinding of EF-G and then GTP hydrolysis should have little effect on the transition from State $\mathrm{H}$ to State $\mathrm{H} 1$ and have no effect on other transitions.

By comparison, we consider the reaction scheme and free-energy landscapes of the translocation proposed by
Kim et al. [11] (see Additional file 1: Figure S1), where it was argued that in the presence of EF-G.GTP the observed transition from the hybrid state to the classical non-rotated state occurs after the dissociation of EF-G but before the re-binding of EF-G. As it will be shown as follows, the theoretical data obtained from the reaction scheme proposed by Kim et al. [11] (see Additional file 1: Figure S1) are inconsistent with the smFRET data [11]. From the reaction scheme proposed by Kim et al. [11] (see Additional file 1: Figure S1), the ratio $(R)$ of the transition of State G to State C to the transition of State $\mathrm{G}$ to State I can be calculated by using Scheme 6, where [EF-G] is the concentration of EF-G.GTP.

From Scheme 6, we easily obtain

$$
R=\frac{k_{H C}}{k_{G I}} \frac{k_{G H}}{\left(k_{H C}+k_{H G}[\text { EF-G] })\right.}
$$

The available biochemical data gave $k_{H G}>100 \mu \mathrm{M}^{-1} \mathrm{~s}^{-1}$ [21], implying that at high [EF-G] $(\geq 0.2 \mu \mathrm{M}), k_{H G}$ [EF-G] $>>k_{H C}\left(1.9 \pm 0.5 \mathrm{~s}^{-1}\right)$. Thus, Eq. (8) can be approximately rewritten as

$$
R=\frac{k_{H C}}{k_{G I}} \frac{k_{G H}}{k_{H G}[\mathrm{EF}-\mathrm{G}]}
$$

The number $(N)$ of the transition from the hybrid state to the classical non-rotated state is thus calculated by $N=1+R$, with $R$ [Eq. (9)] being approximately inversely proportional to [EF-G]. For example, from $N=2.3$ $(R=1.3)$ at $[\mathrm{EF}-\mathrm{G}]=1 \mu \mathrm{M}$ (see Table One in Kim et al. [11] or Figure S7 in Kim et al. [11]), with Eq. (9) we obtain $N=3.6(R=2.6)$ and $7.5(R=6.5)$ at $[\mathrm{EF}-\mathrm{G}]=$ $0.5 \mu \mathrm{M}$ and $0.2 \mu \mathrm{M}$, respectively, which are far away from the smFRET data of $N=2.9$ and 4.8 at [EF-G] = $0.5 \mu \mathrm{M}$ and $0.2 \mu \mathrm{M}$, respectively (see Table One in Kim et al. [11] or Figure S7 in Kim et al. [11]). Moreover, from Eq. (9) it is deduced that even in the range of saturating [EF-G], the number of the transition from the hybrid state to the classical non-rotated state is sensitive to $[E F-G]$, i.e., is inversely proportional to [EF-G]: for example, increasing [EF-G] by 10-fold decreases approximately the number of the transition by 10 -fold. This is

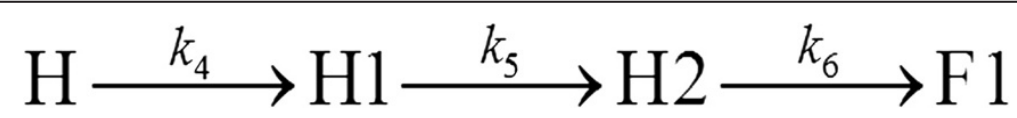

Scheme 4 The transitions during the time period of high FRET efficiency in the first round of intersubunit rotations for the mRNA containing the downstream secondary structure. 


\section{$\mathrm{F} 2 \stackrel{k_{8}}{\longrightarrow} \mathrm{F} 3 \stackrel{k_{9}}{\longrightarrow} \mathrm{H} 1 \stackrel{k_{5}}{\longrightarrow} \mathrm{H} 2 \stackrel{k_{6}}{\longrightarrow} \mathrm{F} 1$}

Scheme 5 The transitions during the time period of high FRET efficiency in the other rounds of intersubunit rotations for the mRNA containing the downstream secondary structure.

contrary to the deduction from the smFRET data [11]. By contrast, in our model of Figure 1, at saturating [EFG] the number of the transition from the hybrid state to the classical non-rotated state is independent of [EF$\mathrm{G}]$, which is consistent with the deduction from the smFRET data [11]. Moreover, the smFRET data at nonsaturating EF-G.GTP can also be quantitatively explained with our model (see Additional file 1: Text S1 and Figures S3 and S4).

In our previous work [27], based on the model similar to that of Figure 1 we have provided quantitative explanations of the intriguing single molecule optical trapping data of $\mathrm{Qu}$ et al. [9] on the rate of ribosome translation through the duplex region of mRNA versus the external force to unzip the duplex. The reduced rate of the translation through the duplex region is induced by the occurrence of the futile transition, which is induced by the resistance force resulting from the unwinding of the downstream mRNA duplex to impede the mRNA translocation. With the same model as shown in Figure 1, the smFRET data of Chen et al. [10] on the effect of the downstream mRNA secondary structure on the tRNA translocation in the 50S subunit and tRNA dissociation from the E site have also been quantitatively explained [12]. The slow dissociation of tRNA from the E site derives also from the occurrence of the futile transition and the dissociation has no effect on the translation rate. Here, with the model shown in Figure 1, we study the dynamics of the multiple intersubunit rotations occurring before the EF-G-catalyzed ribosomal translocation through the mRNA containing the downstream secondary structure, providing quantitative explanations of the smFRET data of Kim et al. [11]. We show that the multiple intersubunit rotations are also induced by the occurrence of the futile transition. By contrast, with the mRNA lacking the downstream mRNA structure, no such resistance force is present and thus, no futile transition occurs, resulting in no occurrence of the transition of the hybrid state to non-rotated state before undergoing translocation. All these quantitative agreements between the theoretical data and the various experimental data from different researchers [9-11] give a strong support to the occurrence of the futile transition during the translocation through the mRNA secondary structure.

Moreover, the multiple fluctuations between the rotated and non-rotated states occurs with EF-G still bound to the ribosome imply that both the rotated and non-rotated states can be bound with EF-G. This is consistent with the recent observations of Chen et al. [19] by using single molecule fluorescence with zero-mode waveguides to directly correlate the ribosome conformation and composition, showing that EF-G can sample both the ribosome conformations.

\section{Conclusions}

Based on our proposed model (Figure 1), we give quantitative explanations of the experimental data of Kim et al. [11] on dynamics of multiple intersubunit rotations occurring before undergoing translocation through the mRNA containing the downstream secondary structures at saturating concentration of EF-G.GTP. The good quantitative agreement between the theoretical data and the smFRET data supports the model showing that at saturating EF-G.GTP the multiple intersubunit rotations occur with EF-G still bound to the ribosome, rather than occur after the release of EF-G from the hybrid state and before the re-binding of EF-G; the multiple intersubunit rotations are induced by the occurrence of the futile transition, which is induced by the resistance force resulting from the unwinding of the downstream mRNA structure to impede the mRNA translocation. Finally, in order to further test the model (Figure 1), we suggest increasing EF-G.GTP concentration to, e.g., $100 \mu \mathrm{M}$ in the smFRET experiment. Under this condition, model of Figure 1 predicts that there are still about $50 \%$ of the traces which are fluctuating before undergoing translocation through the mRNA containing the stem loop. By contrast, model of Kim et al. [11] (see Additional file 1: Figure S1) predicts that there is nearly no trace that is fluctuating, i.e., the ribosomal complex samples the hybrid state nearly once before undergoing translocation through the mRNA containing the stem loop, similar to the case

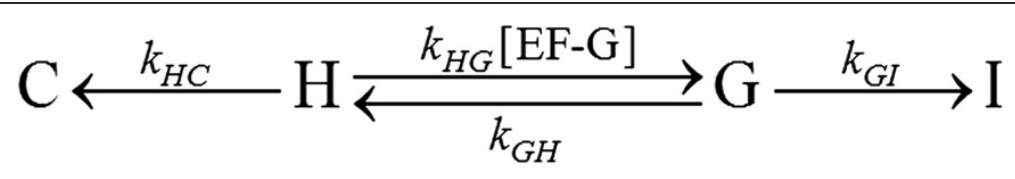

Scheme 6 The transitions for calculation of ratio $R$. 
for translocation through the mRNA lacking the stem loop.

\section{Endnote}

${ }^{a}$ Recent single molecule data showed that the mean time of EF-G.GDP bound to the non-rotated pretranslocation state is longer than that bound to the rotated hybrid state [19]. In addition, the rate of EF-G.GDP releasing from the pretranslocation complex is much smaller than the rate of transition from State F1 to State F2 (see Section entitled "Choice of parameter values"). Thus, for a good approximation, we can neglect the release of EF-G.GDP from State F1.

\section{Additional file}

Additional file 1: Text S1. Time distributions of the high FRET efficiency at different concentrations of EF-G.GTP. Text S2. The derivations of time distributions. Figure $\mathbf{S 1}$. The reaction scheme and free-energy landscapes of the translocation proposed by Kim et al. [S9]. Figure S2. Model of tRNA-mRNA translocation in the ribosome at non-saturating concentration of EF-G.GTP. Figure S3. Results of time distribution of the high FRET efficiency for the mRNA containing the downstream secondary structure (with $P_{E}=0.5$ ) at different concentrations of EF-G.GTP. Figure S4. Results of the time distribution of the high FRET efficiency for the mRNA containing the downstream secondary structure (with $P_{E}=0.5$ ) at $1 \mu \mathrm{M}$ EF-G.GTP. Figure S5. Effects of the uncertainties of the values of the rate constants on the time distributions at saturating EF-G.GTP.

\section{Competing interests}

The author declares that he has no competing interests.

\section{Acknowledgments}

This work was supported by the National Natural Science Foundation of China (Grant No. 11374352).

Received: 4 July 2014 Accepted: 28 October 2014

Published online: 19 November 2014

\section{References}

1. Green R, Noller HF: Ribosomes and translation. Annu Rev Biochem 1997 66:679-716

2. Wintermeyer W, Peske F, Beringer M, Gromadski KB, Savelsbergh A, Rodnina $\mathrm{MV}$ : Mechanisms of elongation on the ribosome: dynamics of a macromolecular machine. Biochem Soc Trans 2004, 32:733-737.

3. Frank J, Gao H, Sengupta J, Gao N, Taylor DJ: The process of mRNA-tRNA translocation. Proc Natl Acad Sci U S A 2007, 104:19671-19678.

4. Shoji S, Walker SE, Fredrick K: Ribosomal translocation: one step closer to the molecular mechanism. ACS Chem Biol 2009, 4:93-107.

5. Petrov A, Kornberg G, O'Leary S, Tsai A, Uemura S, Puglisi JD: Dynamics of the translational machinery. Curr Opin Struct Biol 2011, 21:137-145.

6. Valle M: Almost lost in translation. Cryo-EM of a dynamic macromolecular complex: the ribosome. Eur Biophys J 2011, 40:589-597.

7. Takyar S, Hickerson RP, Noller HF: mRNA helicase activity of the ribosome. Cell 2005, 120:49-58

8. Wen J-D, Lancaster L, Hodges C, Zeri A-C, Yoshimura SH, Noller HF, Bustamante C, Tinoco I Jr: Following translation by single ribosomes one codon at a time. Nature 2008, 452:598-604.

9. Qu X, Wen J-D, Lancaster L, Noller HF, Bustamante C, Tinoco I Jr: The ribosomeuses two activemechanisms to unwind messenger RNA during translation. Nature 2011, 475:118-121.

10. Chen C, Zhang H, Broitman SL, Reiche M, Farrell I, Cooperman BS, Goldman YE: Dynamics of translation by single ribosomes through mRNA secondary structures. Nature Struct Mol Biol 2013, 20:582-588.
11. Kim H-K, Liu F, Fei J, Bustamante C, Gonzalez RL Jr, Tinoco I Jr: A frameshifting stimulatory stem loop destabilizes the hybrid state and impedes ribosomal translocation. Proc Natl Acad Sci U S A 2014, 111:5538-5543.

12. Xie P: Dynamics of tRNA translocation, mRNA translocation and tRNA dissociation during ribosome translation through mRNA secondary structures. Eur Biophys J 2014, 43:229-240.

13. Fischer N, Konevega AL, Wintermeyer W, Rodnina MV, Stark H: Ribosome dynamics and tRNA movement by time-resolved electron cryomicroscopy. Nature 2010, 466:329-333.

14. Lill R, Robertson JM, Wintermeyer W: Binding of the 30-terminus of tRNA to $23 \mathrm{~S}$ rRNA in the ribosomal exit site actively promotes translocation. EMBO J 1989, 8:3933-3938.

15. Feinberg JS, Joseph S: Identification of molecular interactions between P-site tRNA and the ribosome essential for translocation. Proc Natl Acad Sci U S A 2001, 98:11120-11125.

16. Frank J, Agrawal RK: A ratchet-like inter-subunit reorganization of the ribosome during translocation. Nature 2000, 406:318-322.

17. Zavialov AV, Hauryliuk W, Ehrenberg M: Guaninenucleotide exchange on ribosome-bound elongation factor $\mathrm{G}$ initiates the translocation of tRNAs. J Biol 2005, 4:9.

18. Spiegel PC, Ermolenko DN, Noller HF: Elongation factor $G$ stabilizes the hybrid-state conformation of the 70S ribosome. RNA 2007, 13:1473-1482

19. Chen J, Petrov A, Tsai A, O'Leary SE, Puglisi JD: Coordinated conformational and compositional dynamics drive ribosome translocation. Nature Struct Mol Biol 2013, 20:718-727.

20. Qian J, Xie $P$, Xue X-G, Wang P-Y: Modelling of a DNA packaging motor. Chin Phys B 2009, 18:4852-4864

21. Savelsbergh A, Katunin VI, Mohr D, Peske F, Rodnina MV, Wintermeyer W: An elongation factor $\mathrm{G}$-induced ribosome rearrangement precedes tRNA-mRNA translocation. Mol Cell 2003, 11:1517-1523.

22. Uemura S, Aitken CE, Korlach J, Flusberg BA, Turner SW, Puglisi JD: Realtime tRNA transit on single translating ribosomes at codon resolution. Nature 2010, 464:1012-1017.

23. Xie P: Dynamics of tRNA occupancy and dissociation during translation by the ribosome. J Theor Biol 2013, 316:49-60.

24. Blanchard SC, Kim HD, Gonzalez RL Jr, Puglisi JD, Chu S: tRNA dynamics on the ribosome during translation. Proc Natl Acad Sci U S A 2004, 101:12893-12898.

25. Fei J, Kosuri P, MacDougall DD, Gonzalez RL Jr: Coupling of ribosomal L1 stalk and tRNA dynamics during translation elongation. Mol Cell 2008, 30:348-359.

26. Cornish PV, Ermolenko DN, Noller HF, Ha T: Spontaneous intersubunit rotation in single ribosomes. Mol Cell 2008, 30:578-588.

27. Xie P: Model of ribosome translation and mRNA unwinding. Eur Biophys $J$ 2013, 42:347-354.

\section{doi:10.1186/s13628-014-0012-4}

Cite this article as: Xie: Origin of multiple intersubunit rotations before EF-G-catalyzed ribosomal translocation through the mRNA with a downstream secondary structure. BMC Biophysics 2014 7:12.

\section{Submit your next manuscript to BioMed Central and take full advantage of:}

- Convenient online submission

- Thorough peer review

- No space constraints or color figure charges

- Immediate publication on acceptance

- Inclusion in PubMed, CAS, Scopus and Google Scholar

- Research which is freely available for redistribution 